\title{
Article
}

\section{Wiener index of uniform hypergraphs induced by trees}

\author{
Andrey Alekseevich Dobrynin ${ }^{1, *}$ \\ 1 Sobolev Institute of Mathematics, Siberian Branch of the Russian Academy of Sciences, Novosibirsk, 630090, Russia. \\ * Correspondence: dobr@math.nsc.ru
}

Received: 5 September 2019; Accepted: 23 October 2019; Published: 2 November 2019

\begin{abstract}
The Wiener index $W(G)$ of a graph $G$ is defined as the sum of distances between its vertices. A tree $T$ generates $r$-uniform hypergraph $H_{r, k}(T)$ by the following way: hyperedges of cardinality $r$ correspond to edges of the tree and adjacent hyperedges have $k$ vertices in common. A relation between quantities $W(T)$ and $W\left(H_{r, k}(T)\right)$ is established.
\end{abstract}

Keywords: Tree, hypergraph, Wiener index.

MSC: $05 \mathrm{C} 12$

\section{Introduction}

I

$\mathrm{n}$ this paper we are concerned with undirected connected graphs $G$ with vertex set $V(G)$ and edge set $E(G)$. The degree of a vertex is the number of edges that are incident to the vertex. Degree of a vertex $v$ is denoted by $\operatorname{deg}(v)$. If $u$ and $v$ are vertices of $G$, then the number of edges in a shortest path connecting them is said to be their distance and is denoted by $d_{G}(u, v)$. Distance of a vertex $v$ is the sum of distances from $v$ to all vertices of a graph, $d_{G}(v)=\sum_{u \in V(G)} d(v, u)$. The Wiener index is a graph invariant defined as the sum of distances between all vertices of $G$ :

$$
W(G)=\sum_{u, v \in V(G)} d(u, v)=\frac{1}{2} \sum_{v \in V(G)} d_{G}(v) .
$$

It was introduced as a structural descriptor for tree-like molecular graphs [1]. Details on the mathematical properties and chemical applications of the Wiener index can be found in books [2-7] and reviews [8-13]. A number of articles are devoted to comparing of the index of a graph and its derived graphs such as the line graph, the total graph, thorny and subdivision graphs of various kind (see, for example, [14-17]). Hypergraphs generalize graphs by extending the definition of an edge from a binary to an $r$-ary relation. Wiener index of some classes of hypergraphs was studied in [18-20]. Chemical applications of hypergraphs were discussed in [21,22].

Define a class of $r$-uniform hypergraphs $H_{r, k}(T)$ induced by $n$-vertex trees $T$. Edges of a tree correspond to hyperedges of cardinality $r$ and adjacent hyperedges have $k$ vertices in common, $1 \leq k \leq\lfloor r / 2\rfloor$. Examples of a tree and the corresponding hypergraph are shown in Figure 1. The number of vertices of $H_{r, k}(T)$ is equal to $(n-2)(r-k)+r$. We are interesting in finding a relation between quantities $W(T)$ and $W\left(H_{r, k}(T)\right)$.

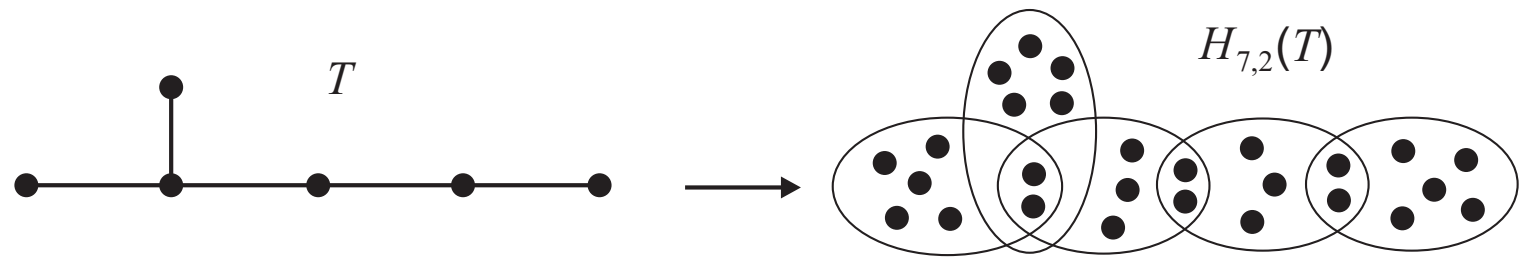

Figure 1. Tree $T$ and the induced hypergraph $H_{7,2}(T)$. 


\section{Main result}

Wiener indices of a tree and its induced hypergraph satisfy the following relation.

Theorem 1. For the induced hypergraph $H_{r, k}(T)$ of a tree $T$ with $n$ vertices,

$$
W\left(H_{r, k}(T)\right)=(r-k)^{2} W(T)+n\left(\begin{array}{l}
k \\
2
\end{array}\right)-(n-1)\left(\begin{array}{c}
r-2 k+1 \\
2
\end{array}\right) .
$$

This result may be useful for ordering of Wiener indices of hypergraphs. If $r$ and $k$ are fixed, then the ordering of the Wiener index of induced hypergraphs $H_{r, k}$ for $n$-vertex trees is completely defined by the ordering of the index of trees. In particular,

$$
W\left(H_{r, k}\left(S_{n}\right)\right) \leq W\left(H_{r, k}(T)\right) \leq W\left(H_{r, k}\left(P_{n}\right)\right)
$$

for any $n$-vertex tree $T$, where $S_{n}$ and $P_{n}$ are the star and the path with $n$ vertices,

$$
\begin{gathered}
W\left(H_{r, k}\left(S_{n}\right)\right)=(r(n-1)[2 n(r-2 k)+8 k-3 r-1]+k(n-2)[k(2 n-3)+1]) / 2, \\
W\left(H_{r, k}\left(P_{n}\right)\right)=n(r-k)\left[(r-k) n^{2}+10 k-4 r-3\right] / 6+2 k^{2}-k(2 r+1)+r(r+1) / 2 .
\end{gathered}
$$

\section{Proof of Theorem 1}

The edge subdivision operation for an edge $(x, y) \in E(G)$ is the deletion of $(x, y)$ from graph $G$ and the addition of two edges $(x, v)$ and $(v, y)$ along with the new vertex $v$. Vertex $v$ is called the subdivision vertex. Denote by $T_{e}$ the tree obtained from the subdivision of edge $e$ in a tree $T$. The distance $d_{G}(v, U)$ from a vertex $v \in V(G)$ to a vertex subset $U \subseteq V(G)$ is defined as $d_{G}(v, U)=\sum_{u \in U} d_{G}(v, u)$.

Lemma 2. Let $T_{e_{1}}, T_{e_{2}}, \ldots, T_{e_{n-1}}$ be trees obtained by subdivision of edges $e_{1}, e_{2}, \ldots, e_{n-1}$ of $n$-vertex tree $T$ with subdivision vertices $v_{1}, v_{2}, \ldots, v_{n-1}$, respectively. Then

$$
d_{T_{e_{1}}}\left(v_{1}\right)+d_{T_{e_{2}}}\left(v_{2}\right)+\cdots+d_{T_{e_{n-1}}}\left(v_{n-1}\right)=2 W(T) .
$$

Proof. Let $v$ be the subdivision vertex of edge $e=(x, y)$ of a tree $T$. Denote by $V_{x}$ and $V_{y}$ the sets of vertices of two connected components after deleting edge $e$ from $T$ where $x \in V_{x}$ and $y \in V_{y}$. Since $d_{T}(x)=d_{T}\left(x, V_{x}\right)+$ $\left|V_{y}\right|+d_{T}\left(y, V_{y}\right)$ and $d_{T}(y)=d_{T}\left(y, V_{y}\right)+\left|V_{x}\right|+d_{T}\left(x, V_{x}\right), d_{T}\left(x, V_{x}\right)+d_{T}\left(y, V_{y}\right)=\left(d_{T}(x)+d_{T}(y)-n\right) / 2$. Then

$$
\begin{aligned}
d_{T_{e}}(v) & =\sum_{u \in V_{x}}\left[d_{T_{e}}(v, x)+d_{T}(x, u)\right]+\sum_{u \in V_{y}}\left[d_{T_{e}}(v, y)+d_{T}(y, u)\right] \\
& =d_{T}\left(x, V_{x}\right)+d_{T}\left(y, V_{y}\right)+n=\left(d_{T}(x)+d_{T}(y)+n\right) / 2 .
\end{aligned}
$$

Klein et al. [23] proved that $\sum_{v \in V(T)} \operatorname{deg}(v) d_{T}(v)=4 W(T)-n(n-1)$ for an arbitrary $n$-vertex tree $T$. Then

$$
\begin{aligned}
2 \sum_{i=1}^{n-1} d_{T_{e_{i}}}\left(v_{i}\right) & =\sum_{(x, y) \in E(T)}\left(d_{T}(x)+d_{T}(y)+n\right) \\
& =\sum_{v \in V(T)} \operatorname{deg}(v) d_{T}(v)+n(n-1)=4 W(T) .
\end{aligned}
$$

For convenience, we assume that pendent hyperedges are also adjacent with fictitious hyperedges shown by dashed lines in Figure 2. Denote by $B_{i}, i=1,2, \ldots n$, the vertices of a hypergraph $H=H_{r, k}(T)$ belonging to hyperedge intersections and let $A=V(H) \backslash B_{1} \cup B_{2} \cup \cdots \cup B_{n}$. We assume that edge $E_{i}$ of the induced 


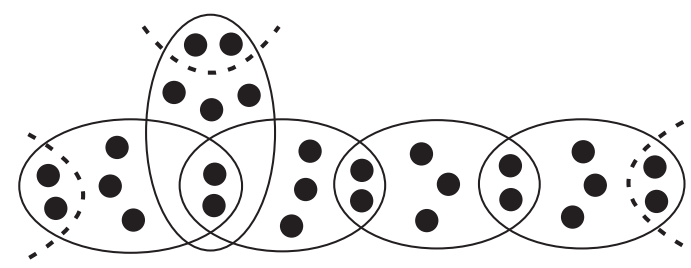

Figure 2. The hyperedges shown by dashed lines

hypergraph corresponds to edge $e_{i}$ of the source tree $T, i=1,2, \ldots, n-1$. Let $d_{G}(U)=\sum_{u \in U} d_{G}(u)$ for $U \subseteq V(G)$. Then the Wiener index of $H$ can be represented as follows:

$$
W(H)=\frac{1}{2}\left(\sum_{i=1}^{n-1} d_{H}\left(E_{i} \cap A\right)+\sum_{i=1}^{n} d_{H}\left(B_{i}\right)\right) .
$$

Let $u \in E_{i} \cap A$ and $v_{i}$ be the subdivision vertex of edge $e_{i}$ in $T, i=1,2, \ldots, n-1$. Then

$$
\begin{aligned}
d_{H}(u)= & (r-2 k-1)+k+k+2(r-k)+\cdots+2(r-k)+3(r-k)+\cdots+3(r-k)+\ldots \\
= & (r-2 k-1)-2(r-2 k)+(r-k)+(r-k)+2(r-k)+\cdots+2(r-k) \\
& +3(r-k)+\cdots+3(r-k)+4(r-k)+\cdots+4(r-k)+\ldots \\
= & (r-k) d_{T_{e_{i}}}\left(v_{i}\right)-2(r-2 k)+(r-2 k-1) .
\end{aligned}
$$

Summing this equality for all vertices of intersection $E_{i} \cap A$, we have $d_{H}\left(E_{i} \cap A\right)=(r-2 k) d_{H}(u)=$ $(r-2 k)\left[(r-k) d_{T_{e_{i}}}\left(v_{i}\right)-(r-2 k+1)\right]$. Applying Lemma 2, we can write

$$
\begin{aligned}
\sum_{i=1}^{n-1} d_{H}\left(E_{i} \cap A\right) & =(r-2 k)\left((r-k) \sum_{i=1}^{n-1} d_{T_{e_{i}}}\left(v_{i}\right)-(n-1)(r-2 k+1)\right) \\
& =(r-2 k)[2(r-k) W(T)-(n-1)(r-2 k+1)] .
\end{aligned}
$$

Let $u \in B_{i}$ and vertex $v_{i}$ of $T$ corresponds to this hyperedge intersection, $i=1,2, \ldots, n$. Then

$$
\begin{aligned}
d_{H}(u) & =(k-1)+(r-k)+\cdots+(r-k)+2(r-k)+\cdots+2(r-k)+3(r-k)+\cdots+3(r-k)+\cdots \\
& =(r-k) d_{T}\left(v_{i}\right)+(k-1) .
\end{aligned}
$$

Summing this equality for all vertices of the hyperedge intersection $B_{i}$, we have $d_{H}\left(B_{i}\right)=k d_{H}(u)=$ $k\left[(r-k) d_{T}\left(v_{i}\right)+(k-1)\right]$. For vertices of all intersections,

$$
\sum_{i=1}^{n} d_{H}\left(B_{i}\right)=k\left[(r-k) \sum_{i=1}^{n} d_{T}\left(v_{i}\right)+n(k-1)\right]=2 k(r-k) W(T)+n k(k-1) .
$$

Substitution expressions (2) and (3) back into Equation (1) completes the proof.

Acknowledgments: This work is supported by the Russian Foundation for Basic Research (project numbers 19-01-00682 and 17-51-560008).

Conflicts of Interest: "The author declare no conflict of interest."

\section{References}

[1] Wiener, H. (1947). Structural determination of paraffin boiling points. Journal of the American Chemical Society, 69(1), 17-20.

[2] Balaban, A. T., Motoc, I., Bonchev, D., \& Mekenyan, O. (1983). Topological indices for structure-activity correlations. In Steric effects in drug design (pp. 21-55). Springer, Berlin, Heidelberg.

[3] Gutman, I., \& Furtula, B. (Eds.) (2012). Distance in Molecular Graphs Theory. Mathematical chemistry monographs, 12, Univ. Kragujevac, Kragujevac, Serbia. 
[4] Gutman, I., \& Furtula, B. (Eds.) (2012). Distance in Molecular Graphs Applications. Mathematical chemistry monographs, 13, Univ. Kragujevac, Kragujevac, Serbia.

[5] Gutman, I., \& Polansky, O. E. (1986).Mathematical Concepts in Organic Chemistry, Springer-Verlag, Berlin.

[6] Todeschini, R., \& Consonni, V. (2008). Handbook of molecular descriptors (Vol. 11). John Wiley \& Sons.

[7] Trinajstić, N. (1992). Chemical Graph Theory. CRC Press, Boca Raton.

[8] Dobrynin, A. A., Entringer, R., \& Gutman, I. (2001). Wiener index of trees: theory and applications. Acta Applicandae Mathematica, 66(3), 211-249.

[9] Dobrynin, A. A., Gutman, I., Klavžar, S., \& Žigert, P. (2002). Wiener index of hexagonal systems. Acta Applicandae Mathematica, 72(3), 247-294.

[10] Nikolić, S., \& Trinajstić, N. (1995). The Wiener index: Development and applications. Croatica Chemica Acta, 68(1), 105-129.

[11] Entringer, R. C., Jackson, D. E., \& Snyder, D. A. (1976). Distance in graphs. Czechoslovak Mathematical Journal, 26(2), 283-296.

[12] Knor, M., Škrekovski, R., \& Tepeh, A. (2015). Mathematical aspects of Wiener index. Ars Mathematica Contemporanea, 11(2), 327-352.

[13] Entringer, R. C. (1997). Distance in graphs: trees. Journal of Combinatorial Mathematics and Combinatorial Computing , 24, 65-84.

[14] Eliasi, M., Raeisi, G., \& Taeri, B. (2012). Wiener index of some graph operations. Discrete Applied Mathematics, 160(9), 1333-1344.

[15] Gutman, I. (1998). Distance of thorny graphs. Publications de l'Institut Mathématique (Beograd), 63(31-36), 73-74.

[16] Knor, M., \& Škrekovski, R. (2014). Wiener index of line graphs. In Quantitative Graph Theory: Mathematical Foundations and Applications, 279-301. 279-301.

[17] Dobrynin, A. A., \& Mel'nikov, L. S. (2012). Wiener index of line graphs. In: Distance in Molecular Graphs Theory, Gutman, I., \& Furtula, B. (Eds.). Univ. Kragujevac, Kragujevac, Serbia, 85-121.

[18] Guo, H., Zhou, B., \& Lin, H. (2017). The Wiener index of uniform hypergraphs. MATCH Communications in Mathematical and in Computer Chemistry, 78, 133-152.

[19] Rani, L. N., Rajkumari, K. J., \& Roy, S. (2019). Wiener Index of Hypertree. In Applied Mathematics and Scientific Computing (pp. 497-505). Birkhäuser, Cham.

[20] Sun, L., Wu, J., Cai, H., \& Luo, Z. (2017). The Wiener index of $r$-uniform hypergraphs. Bulletin of the Malaysian Mathematical Sciences Society, 40(3), 1093-1113.

[21] Konstantinova, E. V., \& Skorobogatov, V. A. (2001). Application of hypergraph theory in chemistry. Discrete Mathematics, 235(1-3), 365-383.

[22] Konstantinova, E. (2000). Chemical hypergraph theory. Lecture Notes from Combinatorial \& Computational Mathametics Center, http://com2mac. postech. ac. kr.

[23] Klein, D. J., Mihalić, Z., Plavšić, D., \& Trinajstić, N. (1992). Molecular topological index: A relation with the Wiener index. Journal of Chemical Information and Computer Sciences, 32(4), 304-305.

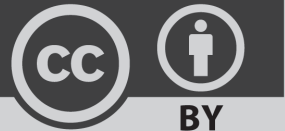

BY
(C) 2019 by the authors; licensee PSRP, Lahore, Pakistan. This article is an open access article distributed under the terms and conditions of the Creative Commons Attribution (CC-BY) license (http://creativecommons.org/licenses/by/4.0/). 